2. R. Montague, Semantical closure and non-finite axiomatizability. I, Proceedings of the 1959 International Symposium on the Foundations of Mathematics: Infinitistic Methods, to appear.

3. K. Gödel, The consistency of the continuum hypothesis, Princeton, N. J., Princeton University Press, 1953.

4. I. L. Novak, $A$ construction for models of consistent systems, Fund. Math. vol. 37 (1950) pp. 87-110. 21-28.

5. J. R. Shoenfield, A relative consistency proof, J. Symb. Logic vol. 19 (1954) pp.

6. A. Tarski, A. Mostowski and R. M. Robinson, Undecidable theories, Amsterdam, North-Holland Publishing Co., 1953.

University of California, Berkeley

\title{
SOLUTION TO A PROBLEM OF ROSE AND ROSSER
}

ATWELL R. TURQUETTE

In a recent article by Rose and Rosser [1], the question is raised concerning the possibility of proving the following theorem using only the first three of Eukasiewicz' axioms for infinite-valued logic together with his rules of inference [2]:

$$
C C Q P C Q R \equiv C C P Q C P R \text {. }
$$

The question is not only interesting in itself, but sheds some light on problems of independence relating to Lukasiewicz' axioms. For example, in another recent paper [3], C. A. Meredith establishes the dependence of Eukasiewicz' fourth axiom, using only the first three of ' uukasiewicz' axioms together with Rose and Rosser's Theorem 3.51.

The purpose of this paper is to establish a negative answer to the Rose-Rosser question. This will be done in a way which will illustrate the use of many-valued logics [4] as instruments for deciding questions of independence, and from this, one will be able to see that in deciding a negative answer to the Rose-Rosser question, a logic with at least four truth-values is required. To this end, let $A P Q$ be defined as $C C P Q Q$ and consider the following axiom schemes and rule of inference:

Axiom schemes:

A1. $C P C Q P$.

A2. CCPQCCQRCPR.

A3. $C A P Q A Q P$.

Received by the editors May 17, 1960. 
Rule of inference:

R1. If $P$ and $C P Q$, then $Q$.

A1, A2, and A3 are the first three axioms introduced by Eukasiewicz for infinite-valued logic, and since axiom schemes are being used, there is no need for a rule of substitution in addition to R1. A yields sign " $\vdash$ " may now be defined in the usual manner in terms of $\mathrm{A} 1, \mathrm{~A} 2, \mathrm{~A} 3$, and $\mathrm{R} 1$. Let $c(p, q)$ denote the truth-value function which is associated with a statement of the form $C P Q$, and let the values of $c(p, q)$ be defined by the following matrix:

\begin{tabular}{c|cccc}
$c(p, q)$ & 1 & 2 & 3 & 4 \\
\hline 1 & 1 & 2 & 3 & 4 \\
2 & 1 & 1 & 1 & 3 \\
3 & 1 & 3 & 1 & 3 \\
4 & 1 & 3 & 1 & 1
\end{tabular}

By means of the matrix $T$, the axiomatic system based on A1, A2, $\mathrm{A} 3$, and R1 may be interpreted as a 4-valued statement calculus with one designated truth-value in the sense of Rosser and Turquette. (See Chapters II and III of Many-valued logics.) It is then easy to establish the following results:

Theorem 1. If the designated truth-value is 1 and $\vdash \mathrm{X}$, then $\mathrm{X}$ takes the designated truth-value exclusively-i.e., the axiomatic stipulation based on $\mathrm{A} 1, \mathrm{~A} 2, \mathrm{~A} 3$, and $\mathrm{R} 1$ is plausible with respect to the truth-value stipulation determined by $M=4, S=1$, and the matrix T (see Manyvalued logics, pp. 27-28 and p. 34).

Proof. By applying a standard truth-table method of calculation making use of the matrix $T$, it can be shown that each of the axioms A1, A2, A3 takes the truth-value 1 exclusively, and that the rule of inference $\mathrm{R} 1$ is valid.

THEOREM 2. Not-トACPQCQP-i.e., Lukasiewicz' fourth axiom for infinite-valued logic is not provable using A1, A2, A3, and R1 alone.

Proof. By definition, $A C P Q C Q P$ is $C C C P Q C Q P C Q P$. A truthtable check by means of $T$ will show that this axiom takes the undesignated value 3 when $P$ takes the value 2 and $Q$ takes the value 4 . Hence, Theorem 2 follows from Theorem 1.

THEOREM 3. Rose and Rosser's 3.51 is not provable using only A1, $\mathrm{A} 2, \mathrm{~A} 3$, and R1-i.e., the answer to the Rose-Rosser question is negative. 
Proof. If 3.51 were provable using only $\mathrm{A} 1, \mathrm{~A} 2, \mathrm{~A} 3$ and $\mathrm{R} 1$, then by Meredith's proof mentioned above which establishes the dependence of Eukasiewicz' fourth axiom for infinite-valued logic, it would be possible to get $\vdash A C P Q C Q P$. Since this is contradictory to Theorem 2, Theorem 3 follows at once.

When the present author first solved the Rose-Rosser problem, use was made of a 6-valued logic with two designated truth-values to establish Theorems 1, 2, and 3. The 6-valued logic was based on a 6 by 6 matrix very similar in structure to T. The solution was communicated to Professor Rosser who coded and checked the most difficult truth-table calculations for the 6-valued logic on an electronic computer. After verifying the desired results for 6-valued logic, Rosser suggested that it should be possible to use a 4 by 4 matrix. The choice of the matrix $T$ is in line with his suggestion. That no further such reduction can be made in the number of truth-values for the logic used in establishing Theorems 1,2 , and 3 , may be seen by checking all possible 3 by 3 and 2 by 2 matrices. Fortunately, the number of cases required for such a check can be greatly reduced by capitalizing on the requirements specified in the proofs of Theorems 1 and 2.

\section{BIBLIOGRAPHY}

1. Alan Rose and J. B. Rosser, Fragments of many-valued statement calculi, Trans. Amer. Math. Soc. vol. 87 (1958) pp. 1-53.

2. Jan Lukasiewicz and Alfred Tarski, Untersuchungen über den Aussagenkalkiul, Comptes Rendus des Séances de la Société des Sciences et des Lettres de Varsovie, Class III, vol. 23 (1930) pp. 30-50. For an English translation of this, see Alfred Tarski's Logic, semantics, metamathematics, Oxford, University Press, 1956, pp. 38-59.

3. C. A. Meredith, The dependence of an axiom of Lukasiewicz, Trans. Amer. Math. Soc. vol. 87 (1958) p. 54.

4. J. B. Rosser and A. R. Turquette, Many-valued logics, Amsterdam, NorthHolland, 1952.

UNIVERSITY OF ILLINOIS 